\title{
Robotic surgery; into the era of common place
}

\author{
Yong Beom Kim ${ }^{1,2}$ \\ ${ }^{1}$ Department of Obstetrics and Gynecology, College of Medicine, Seoul National University, Seoul; '2Department of Obstetrics and Gynecology, \\ Seoul National University Bundang Hospital, Seongnam, Korea
}

Since the past few decades, robot-assisted laparoscopy becomes an advancement of traditional laparoscopy and allows more complex procedures in many fields of surgery through main advantages including improved visualization by three-dimensional view, better ergonomics, no tremor, and shorter learning curves. Although the evidence from large-scaled randomized controlled trials in gynecology are lacking, gynecologic robot-assisted laparoscopy has shown exponential growth and has been progressively implemented as the standard procedure in various gynecologic field [1].

Recently, the US Food and Drug Administration cautioned against the use of robotic surgery in cancer operations, stating that "survival benefits to patients when compared to traditional surgery have not been established" after phase III Laparoscopic Approach to Cervical Cancer (LACC) trial. This phase III trial was designed for the definitive comparison of survival outcomes of radical hysterectomy in patients with early stage cervical cancer between minimally invasive surgery (MIS) and open procedure. This trial demonstrated that MIS is associated with lower rates of disease-free survival and overall survival than open procedure [2]. The findings of this LACC trial are consistent with those of a retrospective study based on the data from the Surveillance, Epidemiology, and End Results (SEER) program database of National Cancer Database (NCDB) for the year of 2000-2010 period [3].

Even though underlying mechanism that causes the poorer outcome of MIS in cervical cancer has yet to be identified, the collective findings have largely halted MIS for cervical cancer. Therefore, several societies announced position statement for MIS in cervical cancer. Korean Society of Gynecologic Oncology (KSGO), one of representative society in Korea, stated that all cervical cancer patients scheduled to undergo radical hysterectomy should be informed about the results of the LACC trial together with institutional data before choosing MIS. Furthermore, KSGO emphasized that establishment of optimal indication for MIS based on the tumor size and surgical methods to minimize tumor destruction or intraperitoneal spillage during colpotomy is required to ensure the oncologic safety of MIS in cervical cancer [4].

On the other hand, a recent systematic review showed that robotic hysterectomy may be a generally safer and better option than open or laparoscopic hysterectomy for patients with endometrial cancer [5]. Another meta-analysis compared robotic and laparoscopic hysterectomy concluded that there was no significant difference in the duration of surgery and the blood loss, the total complication rate and conversions to laparotomy were less in the robotic hysterectomy [6]. Unfortunately, however, very limited data are available regarding oncologic outcomes. One retrospec-

- Received: March 22, 2021 • Accepted: March 22, 2021

- Correspondening author: Yong Beom Kim

Department of Obstetrics and Gynecology, Seoul National University Bundang Hospital, 82 Gumi-ro 173beon-gil, Bundang-gu, Seongnam 13620, Korea E-mail:ybkimlh@snubh.org

This is an Open Access article distributed under the terms of the Creative Commons Attribution Non-Commercial License (http://creativecommons.org/ licenses/by-nc/4.0) which permits unrestricted non-commercial use, distribution, and reproduction in any medium, provided the original work is properly cited. 
tive studies showed no significant difference in neither survival nor recurrence comparing with SEER data for early stage endometrial cancer [7].

Hence the assessment of value of MIS is complicated, especially robotics in cancer surgery, much of the early development was performed with evidence of shorter recovery time and lower complication rates, so that one can debate oncological outcomes. The evaluation of new procedures and devices usually starts from small studies to evaluate an efficacy, and then moves to large comparative trials to demonstrate effectiveness compared with a standard therapy. Considering this facts, randomized trials following stepwise processes such as that recommended in the IDEAL (Idea, Development, Exploration, Assessment, Long-term study) framework should be preceded in the development of a new surgical procedure [8,9]. However, direct application of this framework to procedures already in use is not easy as new practice is often applied faster than evidence of data.

Cost-effectiveness of robotic surgery still remains in controversial due to heterogeneous multiple factors such as direct and indirect costs, institutional volume of cases as well as different reimbursement systems along with country by country [10]. Although the cost-effectiveness of robot-assisted procedures has been questioned, application of robot-assisted technology appears to have substantially increased for many years. In addition, robot-assisted surgery has been intensely marketed not only to physicians and hospitals but also directly to patients [11]. Struggling competition among hospitals is also one of important driving forces in the diffusion of robotic surgery [12]. Given this controversy, it has been proposed that manufacturers should reevaluate and reduce the cost of robotic assisted surgery. This reduced cost could help alleviate much of the criticism surrounding the procedures and consequently allow more patients to benefit of MIS without a significant increase of overall costs.

Based on current data and evidences, robot-assisted laparoscopy represents a valuable alternative to laparotomy as well as conventional laparoscopy, and it will overcome the limitations of laparotomy or laparoscopy. Although cost-effectiveness still represents a limitation, robot-assisted surgery has the potential to become cost-effective in high-volume centers while industry could reduce the cost of robotic platform and devices. The most important thing remained is that random- ized clinical trials are needed to define the efficacy in the view of clinical outcome and patients' quality of life.

\section{References}

1. Lee S, Kim MR, Seong SJ, Paek J, Lee YS, Nam EJ, et al. Trends in robotic surgery in Korean gynecology. Gyne Robot Surg 2020;1:506.

2. Ramirez PT, Frumovitz M, Pareja R, Lopez A, Vieira M, Ribeiro R, et al. Minimally invasive versus abdominal radical hysterectomy for cervical cancer. N Engl J Med 2018;379:1895-904.

3. Melamed A, Margul DJ, Chen L, Keating NL, Del Carmen MG, Yang J, et al. Survival after minimally invasive radical hysterectomy for early-stage cervical cancer. N Engl J Med 2018;379:1905-14.

4. Kim M, Kong TW, Kim S, Kim SC, Kim YB, Kim JW, et al. Minimally invasive surgery for radical hysterectomy in women with cervical cancer: Korean Society of Gynecologic Oncology, Korean Society of Obstetrics and Gynecology, and Korean Society of Gynecologic Endoscopy and Minimally Invasive Surgery position statement. J Gynecol Oncol 2019;30:e104.

5. Park DA, Lee DH, Kim SW, Lee SH. Comparative safety and effectiveness of robot-assisted laparoscopic hysterectomy versus conventional laparoscopy and laparotomy for endometrial cancer: a systematic review and meta-analysis. Eur J Surg Oncol 2016;42:1303-14.

6. Ind T, Laios A, Hacking M, Nobbenhuis M. A comparison of operative outcomes between standard and robotic laparoscopic surgery for endometrial cancer: a systematic review and meta-analysis. Int J Med Robot 2017;13:e1851.

7. Kilgore JE, Jackson AL, Ko EM, Soper JT, Van Le L, Gehrig PA, et al. Recurrence-free and 5-year survival following robotic-assisted surgical staging for endometrial carcinoma. Gynecol Oncol 2013;129:49-53.

8. Pennell CP, Hirst AD, Campbell WB, Sood A, Agha RA, Barkun JS, et al. Practical guide to the Idea, development and exploration stages of the IDEAL framework and recommendations. Br J Surg 2016;103:607-15.

9. McCulloch P, Altman DG, Campbell WB, Flum DR, Glasziou P, Marshall JC, et al. No surgical innovation without evaluation: the IDEAL recommendations. Lancet 2009;374:1105-12.

10. Ngô C, Cornou C, Rossi L, Bats AS, Bensaid C, Frati A, et al. Evidence for the use of robotically assisted surgery in gynecologic cancers. Curr Opin Oncol 2016;28:398-403.

11. Schiavone MB, Kuo EC, Naumann RW, Burke WM, Lewin SN, Neugut Al, et al. The commercialization of robotic surgery: unsubstantiated marketing of gynecologic surgery by hospitals. Am J obstet Gynecol 2012;207:174.e1-7.

12. Wright JD, Tergas AI, Hou JY, Burke WM, Chen L, Hu JC, et al. Effect of regional hospital competition and hospital financial status on the use of robotic-assisted surgery. JAMA Surg 2016;151:61220. 